\title{
Caracterización de pacientes con Acromegalia atendidos en la Consulta de Endocrinología del Instituto
}

Salvadoreño del Seguro Social

\author{
Elliana Regina López Estrada \\ Recepción: 03 de septiembre 2012 \\ Aprobado: $\quad 08$ de enero 2013
}

\section{Resumen}

En el Instituto Salvadoreño del Seguro Social (ISSS) a través del consultorio de especialidades, se tienen pocos datos sobre la población con acromegalia, por lo que en el presente estudio se describen las principales características de 40 pacientes con dicha patología atendidos durante el 2010. El rango de edad del grupo osciló entre los 21 y 75 años, siendo en su mayoría mujeres (60\%). Las manifestaciones clínicas encontradas fueron: crecimiento acral y facial, cefalea, trastornos visuales y artralgias; con inicio más frecuente entre la segunda y quinta décadas de la vida. El tiempo promedio de evolución de la enfermedad al diagnóstico fue de 3.8 años, el cual es menor al descrito en la literatura mundial; sin embargo, la mayoría debutó con macroadenomas y extensión perisellar. El 95\% fue abordado con resección quirúrgica inicial y posteriormente la mayoría recibió análogos de somatostatina de larga y corta acción $(27.5 \%$ y $22.5 \%$ respectivamente). Se encontró que el $55 \%$ del grupo no había logrado control bioquímico, siendo más común en mujeres y en presencia de macroadenomas hipofisiarios con algún grado de extensión. El tamizaje de complicaciones cardiovasculares y colorrectales se realizó en $17.5 \%$ y $10 \%$ de pacientes, respectivamente, reportándose anormalidades en la mayoría, incluso

un caso de cáncer colónico avanzado. Se espera que la información obtenida constituya la base para aseguraren la institución la atención óptima y el manejo integral del paciente con acromegalia.

Palabras clave: Acromegalia - Descriptivo - Características tumorales - Manifestaciones clínicas - Ecocardiografía - Colonoscopía - El Salvador.

\section{Introducción}

La acromegalia es una endocrinopatía de lenta evolución, caracterizada por crecimiento desproporcionado tanto de esqueleto, como de órganos y tejidos blandos. Resulta de la producción excesiva de Hormona del Crecimiento $(\mathrm{HC})$ a partir de adenomas hipofisiarios ( $99 \%$ de casos), generando niveles circulantes de HC persistentemente altos 1. La experiencia con acromegalia en América Latina es limitada, los esfuerzos de investigación provienen principalmente de México, Colombia, Brasil y Cuba; y son sobre aspectos puntuales y complicaciones de la patología, así como propuestas de algoritmos para abordaje y manejo regionales, tomando en cuenta las opciones terapéuticas reales con las que se dispone en estos países 2,3,4,5. Los datos demográficos de la enfermedad, provienen de extensos

1.Doctora en medicina, Internista del Instituto Salvadoreño del Seguro Social. elliana_18@hotmail.com 
estudios poblacionales realizados a nivel mundial. En Centro América, Costa Rica tomó la iniciativa de recopilar datos regionales sobre pacientes con acromegalia, lo cual aún es un trabajo en evolución6. Es así como se concibe el presente estudio, con el objetivo de dar respuesta a la necesidad de conocer características propias de los pacientes atendidos en el ISSS, y contribuir a la creación de estadísticas de El Salvador.

\section{Metodología}

\section{Pacientes y definiciones}

Se trabajó con una muestra de 40 pacientes, 16 hombres y 24 mujeres con acromegalia, mayores de 18 años, atendidos de forma regular en el Consultorio de Especialidades del ISSS, durante el 2010; excluyendo los pacientes en los cuales no hubiera acceso a una fuente confiable de datos. El estudio fue aprobado por el Comité de Ética institucional.

Se investigaron aspectos demográficos y clínicos, así como el tiempo de evolución de la enfermedad al momento del diagnóstico. Para la descripción de las manifestaciones clínicas, se consideraron los signos y síntomas más comunes y directamente atribuibles a la patología, referidos por el paciente al momento de la consulta.

El tamaño tumoral al diagnóstico se tomó de los datos del primer estudio de imagen, documentado en el expediente clínico, eligiendo como medida principal el eje de mayor tamaño en centímetros. Se definió macroadenoma a toda neoplasia superior a $1 \mathrm{~cm}$ en cualquiera de sus ejes, y microadenoma si no superaba $1 \mathrm{~cm}$ en sus ejes.

La extensión tumoral se definió por evidencia de crecimiento del tumor hacia el plano superior, lateral, inferior o de forma difusa. Los métodos de imagen considerados para detección tumoral incluyeron, resonancia magnética nuclear, tomografía computarizada y radiografía convencional.
Para diagnóstico bioquímico de acromegalia, se definió una prueba de supresión de Hormona de Crecimiento $(\mathrm{HC})$ con valor superior a $1 \mathrm{ng} / \mathrm{ml}$ dentro de 2 horas posterior a una carga con $75 \mathrm{~g}$ de glucosa oral, o un valor de factor de crecimiento similar a insulina (IGF-1) mayor de lo normal, según datos poblacionales comparables con edad y sexo del paciente. La medición de $\mathrm{HC}$ al azar no se incluyó en el presente estudio como prueba diagnóstica.

Para identificar el tipo de tratamiento específico utilizado por el paciente con acromegalia se le dividió en tratamiento quirúrgico, farmacológico y radioterapeútico. El tratamiento farmacológico incluyó una subdivisión entre análogos de somatostatina de larga duración (ASLD), análogos de somatostatina de corta duración (ASCD), agonistas de dopamina (AD) y antagonistas del receptor de HC (ARHC). Para evaluar el riesgo de complicaciones cardiovasculares y colorrectales, se realizaron estudios ecocardiográficos y la colonoscopía respectivamente, en cualquier momento del curso de la enfermedad.

La respuesta al tratamiento se clasificó según cumplimiento de criterios de control bioquímico o no, en: "Controlado" y "No controlado". Definiéndose como "controlado" a todo paciente con un valor de HC $<1 \mathrm{ng} / \mathrm{ml}$ en 2 horas posterior a una prueba de supresión de glucosa, o un valor normal de IGF-1 de acuerdo a sexo y edad del paciente. El paciente "no controlado" es aquel que no cumple ninguno de los dos criterios antes mencionados, en base al resultado más reciente consignado en el expediente clínico.

\section{Fuentes y Manejo de la información}

Es un estudio descriptivo, transversal. La recolección de datos se obtuvo a partir del listado oficial de pacientes con acromegalia, elaborado por la endocrinóloga encargada en el Consultorio de Especialidades del ISSS, a través de la revisión de expedientes clínicos y excepcionalmente a través de comunicación directa con el paciente, en el período comprendido entre enero a di- 
ciembre del 2010. Se elaboró un consolidado con los datos generales, resultados de estudios de imagen y pruebas bioquímicas de cada paciente. El análisis de los datos se realizó con Excel y SPSS 16.0. Para variables continuas se calculó media aritmética, desviación estándar, mediana y moda, mientras que para variables ordinales se obtuvo porcentajes. La principal limitante para la recolección de información consistió en extravío de expedientes en archivo, datos y reportes de estudios de imagen y laboratorio incompletos o ausentes en anotaciones médicas.

\section{Resultados}

El $60 \%$ de los pacientes eran mujeres. La edad promedio encontrada fue de 53.4 \pm 13.1 años, mediana de 55 y moda de 47, con rangos que oscilaban entre los 21 a 75 años.

La mayoría (70\%) presentaron, inicio de manifestaciones clínicas antes de los 50 años de edad, principalmente entre la segunda y quinta década de la vida, con una media de $38.8 \pm 12.4$ años, moda de 46 y mediana de 40. Sin embargo, en algunos casos fueron tan tempranas como 14 y tan tardías como 66 años.

Usualmente fueron diagnosticados a los 47 años de edad, con una media de $43 \pm 11.7$ y mediana de 45 (Gráfico 1), calculándose un tiempo promedio de evolución de 3.8 años. La mayor parte de casos se detectó entre 1 a 5 años desde el inicio de los síntomas, con moda y mediana de 2 años; $17.5 \%$ fue diagnosticado en el primer año de evolución.

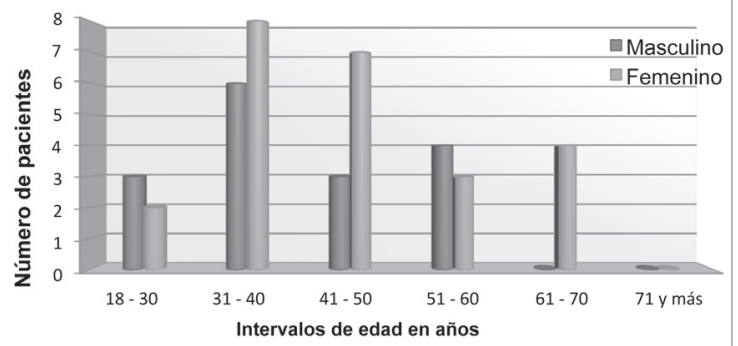

Grafico 1. Distribución de pacientes con acromegalia Según edad al diagnóstico
Dentro de las manifestaciones clínicas más encontradas están: crecimiento acral (62.5\%), alteración de rasgos faciales (50\%), cefalea (32.5\%), anormalidad del campo visual (30\%) y artralgias (27.5\%) (Gráfico 2). Es importante mencionar que la mayoría de pacientes con anormalidad del campo visual por campimetría, no reportaba sintomatología visual como motivo de consulta.

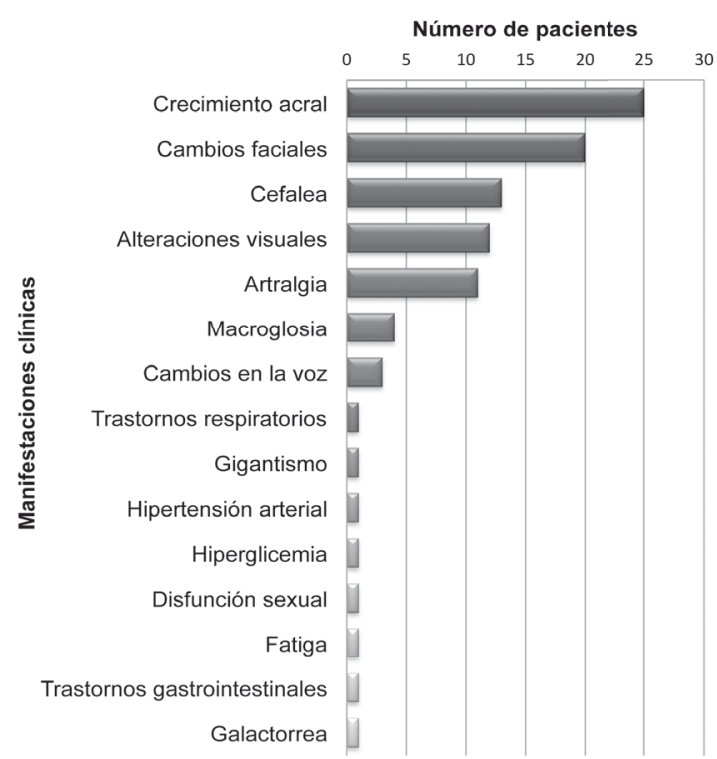

Gráfico 2. Manifestaciones clínicas más frecuentes en pacientes con acromegalia

La etiología de acromegalia en $97.5 \%$ de casos fue un adenoma hipofisiario, en el 2.5 \% restante no se logró identificar la causa, debido a la falta de pruebas genéticas o evidencia de secreción ectópica de hormona de crecimiento. En aquellos pacientes en los que se detectó neoplasia, $76.9 \%$ presentaba un macroadenoma al diagnóstico, $10.3 \%$ microadenoma y en $12.8 \%$ no se encontró informe oficial del tamaño tumoral (Gráfico 3). 


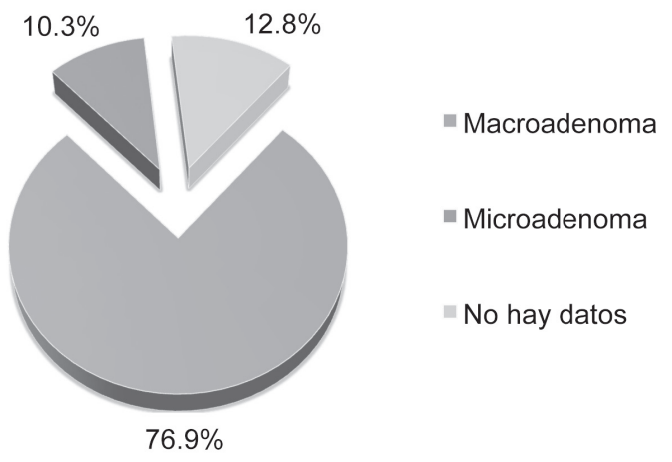

Gráfico 3. Tamaño de neoplasia hipofisiaria identificada según estudio Radiológico al diagnóstico.

Según el eje mayor de la neoplasia se determinó un tamaño promedio de $2.1 \pm 0.8$ cms, mediana de $2.1 \mathrm{cms}$ y moda de 1.6 cms. En $46.1 \%$ de pacientes con adenoma se evidenció extensión perisellar, hacia el plano superior en $20.5 \%$, difusa en $15.4 \%$ y lateral en $10.2 \%$. Ninguno hacia plano inferior (Gráfico 4).

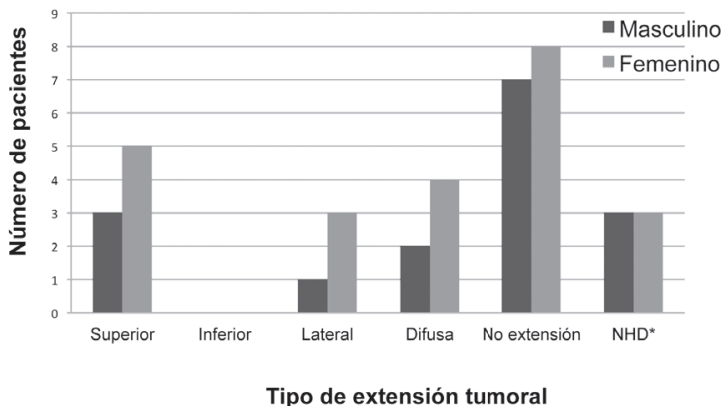

Gráfico 4. Extensión de la neoplasia hipofisiaria fuera de la silla furca al diagnóstico

El estudio de imagen más utilizado para la detección de neoplasia hipofisiaria fue la Resonancia Magnética Nuclear (62.5\%), con menor frecuencia se realizó Tomografía Axial Computarizada $(25 \%)$ y radiografía convencional con énfasis en silla turca (2.5\%).

La prueba de laboratorio más utilizada para el diagnóstico bioquímico de acromegalia fue la medición de HC posterior a prueba de supresión con glucosa $(72.5 \%)$, seguida por medición de IGF-1 en conjunto con test de glucosa (27.5\%). El valor de IGF-1 no se utilizó en ningún caso como única prueba diagnóstica.
En cuanto al tipo de tratamiento se determinó que el $95 \%$ de los pacientes fue abordado inicialmente con cirugía, la vía transesfenoidal fue la más utilizada. El $45 \%$ de los casos recibió radioterapia. Durante el periodo de estudio los fármacos recibidos fueron ASLD (27.5\%), ASCD (22.5\%) y AD (2.5\%). El $30 \%$ de pacientes se encontró sin farmacoterapia, la mayoría por indicación médica después de lograr control bioquímico y en menor medida por suspensión voluntaria.

De acuerdo a las últimas pruebas de laboratorio reportadas en el expediente de cada paciente, el $55 \%$ de ellos se encontró sin un control bioquímico aceptable, $37.5 \%$ había logrado control y de 3 pacientes (7.5\%) no se tenían datos (Tabla 1).

\begin{tabular}{|c|c|c|c|}
\hline \multirow{2}{*}{ Sexo } & \multicolumn{3}{|c|}{ Tabla 1. Estado de control bioquímico } \\
\cline { 2 - 4 } & Controlado & $\begin{array}{c}\text { No } \\
\text { controlado }\end{array}$ & NDD \\
\hline Masculino & 8 & 8 & 0 \\
\hline Femenino & 7 & 14 & 3 \\
\hline Total & 15 & 22 & 3 \\
\hline Porcentaje & $37.5 \%$ & $55.0 \%$ & $7.5 \%$ \\
\hline
\end{tabular}

*NDD: no datos disponibles

De los 22 pacientes identificados sin control bioquímico, la mayoría (63.6\%) eran mujeres, $13.6 \%$ se encontraban sin farmacoterapia y aquéllos bajo tratamiento con fármacos se distribuían en igual número entre los tratados con ASLD y ASCD (36.4\%). El $86.36 \%$ había sido sometido a cirugía, incluso con re intervenciones debido a recidiva tumoral. El $68 \%$ presentaba un macroadenoma al diagnóstico, la mayoría superando $2 \mathrm{~cm}$. en su eje mayor y $45 \%$ mostraba evidencia de extensión perisellar aún tomando en cuenta que el $68.3 \%$ fue diagnosticado dentro de los primeros 5 años de evolución (Tabla 2). 


\begin{tabular}{|c|c|c|c|c|c|}
\hline Sexo & $\begin{array}{l}\text { Tratamiento } \\
\text { farmacológico }\end{array}$ & $\begin{array}{l}\text { Resección } \\
\text { quirúrgica }\end{array}$ & $\begin{array}{l}\text { Tamaño } \\
\text { tumoral }\end{array}$ & $\begin{array}{l}\text { Extensión } \\
\text { tumoral }\end{array}$ & Evolución \\
\hline $\begin{array}{l}\text { M: } 8 \\
(36.4 \%)\end{array}$ & $\begin{array}{l}\text { Octreotide LAR: } \\
8(36.4 \%)\end{array}$ & $\begin{array}{l}\text { Sí: } 19^{*} \\
(86.36 \%)\end{array}$ & $\begin{array}{c}\text { Macro: } \\
15^{* *} \\
(68.2 \%)\end{array}$ & $\begin{array}{l}\text { Sí: } 10^{* * *} \\
(45.45 \%)\end{array}$ & $\begin{array}{l}<1 \text { año: } 4 \\
(18.2 \%)\end{array}$ \\
\hline \multirow[t]{6}{*}{$\begin{array}{l}F: 14 \\
(63.6 \%)\end{array}$} & $\begin{array}{c}\text { Octreotide } \\
\text { diario: } 8 \text { (36.4\%) }\end{array}$ & $\begin{array}{l}\text { No: } 3 \\
\text { (13.64\%) }\end{array}$ & $\begin{array}{l}\text { Micro: } 4 \\
\text { (18.2\%) }\end{array}$ & $\begin{array}{c}\text { No: } 9 \\
(40.90 \%)\end{array}$ & $\begin{array}{l}1 \text { a } 5 \text { años: } \\
11 \text { (50.1\%) }\end{array}$ \\
\hline & Bromocriptina: 0 & & $\begin{array}{c}\text { No } \\
\text { tumor: } 1 \\
(4.5 \%)\end{array}$ & $\begin{array}{l}\text { No hay dato: } \\
2(9.10 \%)\end{array}$ & $\begin{array}{l}6 \text { a } 10 \text { años: } \\
1(4.5 \%)\end{array}$ \\
\hline & $\begin{array}{l}\text { Antagonista de } \\
\text { receptor HC: } 0\end{array}$ & & $\begin{array}{l}\text { No hay } \\
\text { dato: } 2 \\
(9.1 \%)\end{array}$ & $\begin{array}{c}\text { No aplica: } 1 \\
(4.55 \%)\end{array}$ & $\begin{array}{l}11 \text { a } 15 \\
\text { años: } 1 \\
(4.5 \%)\end{array}$ \\
\hline & $\begin{array}{c}\text { No fármaco: } 3 \\
(13.6 \%)\end{array}$ & & & & $\begin{array}{l}16 \text { a } 20 \\
\text { años: } 1 \\
(4.5 \%)\end{array}$ \\
\hline & $\begin{array}{c}\text { No hay dato: } 3 \\
\text { (13.6\%) }\end{array}$ & & & & $>20$ años: 0 \\
\hline & & & & & $\begin{array}{l}\text { No hay } \\
\text { dato: } 4 \\
\text { (18.2\%) }\end{array}$ \\
\hline
\end{tabular}

El tamizaje ecocardiográfico se realizó en 7 pacientes (17.5\%), de los cuales la mayoría (12.5) reportó anormalidad cardiovascular (tabla 3). De forma similar, se hizo colonoscopía en el $10 \%$ de la población, en la cual se encontró lesiones de gravedad como: pólipos, rectitis, colitisinespecífica, divertículos y un caso de cáncer de colon invasivo (Tabla 4).

\begin{tabular}{|l|c|c|c|c|}
\hline \multirow{2}{*}{ Sexo } & \multicolumn{4}{|c|}{ Tabla 3. Resultado de Tamizaje Ecocardiográfico } \\
\cline { 2 - 5 } & Normal & Anormal & $\begin{array}{c}\text { No } \\
\text { realizado }\end{array}$ & NHD* \\
\hline Masculino & 1 & 4 & 11 & 0 \\
\hline Femenino & 1 & 1 & 21 & 1 \\
\hline Total & 2 & 5 & 32 & 1 \\
\hline Porcentaje & $5 \%$ & $12.5 \%$ & $80 \%$ & $2.5 \%$ \\
\hline
\end{tabular}

\begin{tabular}{|l|c|c|c|c|}
\hline \multirow{2}{*}{ sexo } & \multicolumn{4}{|c|}{ Tabla 4. Resultado de Tamizaje Colonoscópico } \\
\cline { 2 - 5 } & Normal & Anormal & $\begin{array}{c}\text { No } \\
\text { realizado }\end{array}$ & NHD* \\
\hline Masculino & 0 & 0 & 16 & 0 \\
\hline Femenino & 0 & 4 & 19 & 1 \\
\hline Total & 0 & 4 & 35 & 1 \\
\hline Porcentaje & $0 \%$ & $10 \%$ & $87.5 \%$ & $2.5 \%$ \\
\hline
\end{tabular}

\section{Discusión}

En la muestra estudiada se encontró similitud con estudios europeos que describen un predominio femenino de la acromegalia $7,8,9,10$, a pesar que mundialmente no se ha observado distinción de sexo, etnia o raza. En cuanto a la edad de diagnóstico, la literatura lo reporta más frecuentemente después de 50 años; esto difiere de lo observado en el Consultorio de Especialidades, ya que en el estudio se encontró que las manifestaciones clínicas y el diagnóstico de la enfermedad se dan a edades mucho más tempranas de la edad promedio esperada, entre la segunda y quinta década de vida, con tiempo de evolución al diagnóstico mucho más corto que el promedio mundial estimado en 10 a 15 años 11,12.

Como se esperaba, casi la totalidad de pacientes presentó como etiología un adenoma hipofisiario, sin embargo, es importante enfatizar que aún con la detección aparentemente temprana del paciente, la mayoría de ellos debutó con un macroadenoma y con algún grado de extensión perisellar. A ese respecto, revisiones internacionales ya han descrito un crecimiento tumoral más rápido y agresivo en jóvenes 11,12, lo que pudiera explicar este hallazgo.

Las manifestaciones clínicas más referidas como causa de consulta fueron aquellas más notorias a simple vista y probablemente de mayor impacto emocional, en especial para el sexo femenino, a la cabeza el excesivo crecimiento de extremidades y alteración de las proporciones facia- 
les, concordando con datos descritos en la literatura 13,14. Aquellos pacientes con cuadros inespecíficos tendieron a ser diagnosticados más tardíamente ya que su sintomatología se asoció con cambios del envejecimiento tal como se ha observado en investigaciones enfocadas en las posibles causas de retraso en el manejo 12,15.

En lo referente al diagnóstico bioquímico de acromegalia en el Consultorio de Especialidades, la mayoría se diagnosticó mediante prueba de supresión con glucosa y en menor porcentaje por una combinación de esta con niveles de IGF-1. Probablemente la medición de IGF-1 no se ha utilizado de forma rutinaria como prueba inicial, dado que actualmente no se cuenta con ella en el laboratorio institucional y es menos conocida.

Según se recomienda en algoritmos terapéuticos internacionales, la gran mayoría de pacientes incluidos en el estudio fue inicialmente abordada por cirugía transesfenoidal, recibiendo posteriormente farmacoterapia y en algunos casos radioterapia. Los pacientes con tratamiento farmacológico durante el periodo de estudio se hallaron recibiendo por igual análogos de somatostatina de corta y larga duración. La literatura actual avala este tipo de fármacos como primera línea, ya que no sólo generan involución tumoral sino también control bioquímico, siendo las formulaciones de acción prolongada las más recomendadas por su comodidad, costo-efectividad y por haber demostrado equivalencia de eficacia con menor frecuencia de aplicaciones 16. El ISSS, por el momento no cuenta con preparados de larga duración en el cuadro básico de medicamentos.

Un punto que merece especial atención es la falta de control bioquímico en más de la mitad de los pacientes estudiados. Es conocido que un mayor tamaño tumoral al diagnóstico, la presencia de extensión perisellar, mayor tiempo de evolución y el compromiso de vasos podrían ser útiles para predecir falla terapéutica 17.
Se observó que de la población sin control bioquímico, la mayor parte es del sexo femenino y se sometieron inicialmente a cirugía, debido a que debutaron con adenomas mayores de $1 \mathrm{~cm}$ en su eje mayor, en algunos casos requiriendo múltiples intervenciones por recidiva tumoral. Cerca de la mitad de dichos pacientes presentaba extensión perisellar al diagnóstico, a pesar que el tiempo de evolución era menor de 5 años.

El tipo de tratamiento farmacológico recibido por la población, al momento del estudio, pareciera no estar relacionado con la falla en el control bioquímico, ya que los pacientes se encontraron utilizando por igual análogos de somatostatina de larga y corta duración, sin embargo, es necesario realizar mayor investigación sobre este punto.

A pesar de su comportamiento benigno, los adenomas hipofisarios con producción excesiva de $\mathrm{HC}$ producen un amplio espectro de morbilidad cardiovascular, respiratoria, endócrina y metabólica 17. Alrededor de un $30 \%$ de pacientes desarrollan disfunción cardiovascular, incluyendo hipertensión, cardiomiopatía, arritmias, hipertrofia de ventrículo izquierdo y deterioro de la función diastólica 18. El resultado de estas complicaciones es una mortalidad, hasta tres veces mayor, en los individuos con acromegalia, que en el resto de la población, y una sobrevida promedio alrededor de 10 años menor. En vista de lo anterior, las guías actuales para el manejo de acromegalia sugieren un estudio ecocardiográfico al diagnóstico en todo paciente, aún sin enfermedad cardíaca conocida 17.

Se identificó que la mayoría de la población con acromegalia, incluida en la presente investigación, no ha sido sometida a estudio ecocardiográfico y se reportaron anormalidades en casi la totalidad de pacientes en los que la evaluación sí se había realizado. Otra complicación de gran importancia es el mayor riesgo de cáncer colorrectal 19,20. Los factores de riesgo en esta población incluyen ser mayor de 50 años de edad, historia familiar de cáncer 
colónico, hipersomatotropismo de más de 10 años de duración, poliposis previa y presencia de más de 6 apéndices cutáneos 21,22. Numerosos estudios muestran una prevalencia de neoplasia colónica de $21 \%$ en el paciente acromegálico, comparado con $9 \%$ en la población general (RR: 2.36) y su aparecimiento se ha observado en individuos jóvenes también, independientemente de la dieta, la raza o la edad de diagnóstico 23.

Todos los pacientes del Consultorio de Especialidades en quienes se realizó colonoscopía, mostraron algún tipo de lesión y existe aún un $87.5 \%$ de la población que no cuenta con este estudio, por lo que se hace necesario incluir en los protocolos institucionales de manejo la realización rutinaria de este tipo de tamizaje.

Finalmente, es evidente que el conocimiento de características generales de la población con acromegalia, es apenas el primer paso si se pretende lograr un mejor manejo de esta patología; en especial, reconociendo que la óptima atención de dichos casos debe ser integral y multidisciplinaria. Es necesario crear bases de datos a nivel institucional, nacional y regional que permitan establecer los fundamentos de mejoras significativas en beneficio de los pacientes.

\section{Agradecimientos}

Al invaluable apoyo del Dr. Max Molina Barriere, Jefe del Departamento de Endocrinología, Consultorio de Especialidades ISSS; Dra. Rosa Lorena Alvarenga, Jefa del Departamento de Medicina Interna ISSS y Dra. Alma Rosa Monterrosa, endocrinóloga a cargo de la consulta de acromegalia del Consultorio de Especialidades.

\section{Fuentes consultadas}

- Cordero, R., et al. Current diagnosis of acromegaly. Endocr Metab Disord 2008; 9:13-19.

- Barkan, A., et al. Management of acromegaly in Latin America: expert panel Recommendations. Pituitary. 2010; 13:168-175.

- Espinosa-de-los-Monteros, A., et al. Clinical and biochemical characteristics of acromegalic patients with different abnormalities in glucose metabolism. Pituitary. 2011; 14:231-235.

- Rosario, P., Frequency of acromegaly in adults with diabetes or glucose intolerance and estimated prevalence in general population. Pituitary 2011; 14: 217-221.

- López, O., et al. Cirugía transesfenoidal: primera opción de tratamiento para adenomas hipofisarios secretores de GH. Rev Cubana Endocrinol. (revista en la internet). 2004; vol.15 n.3

- Chih, H., et al. Guías para el diagnóstico y tratamiento de acromegalia, prolactinomas y enfermedad de Cushing. Acta méd. Costarric. 2004; v.46 supl. 1, pp. $25-36$.

- Nabarro, JD. Acromegaly. Clin Endocrinol. 1987; 26:481-512.

- $\quad$ Ezzat, S., et al. Acromegaly. Clinical and biochemical features in 500 patients. Medicine (Baltimore). 1994; 73: 233-40

- $\quad$ Etxabe, J., et al. Acromegaly: an epidemiological study. J Endocrinol Invest. 1993; 16(3):181-187.

- Mestron, A., et al. Epidemiology, clinical characteristics, outcome, morbidity and mortality in acromegaly based on the Spanish Acromegaly Registry (Registro Español de Acromegalia, REA). Eur J Endocrinol. 1993; 151(4):439-446.

- Melmed, S. Medical progress: acromegaly. N Engl J Med. 2006; 355: 2558-2573.

- $\quad$ Reid, T.J., et al. Features at diagnosis of 324 patients with acromegaly did not change from 1981 to 2006; acromegaly remains under-recognized and under-diagnosed. Clin Endocrinol (Oxf). 2010; 72:203-208.

- Melmed, S. Acromegaly pathogenesis and treatment. J Clin Invest. 2009; 119(11):3189-3202.

- Molitch, M. Clinical manifestations of acromegaly. Endocrinol Metab Clin North Am. 1992; 21:597-614.

- Psaras, T., et al. Demographic factors and the presence of comorbidities do not promote early detection of Cushing's disease and acromegaly. Exp Clin Endocrinol Diabetes. 2011; 119(1): 21-25.

- Murray, R.D., et al. A critical analysis of clinically available somatostatin analog formulations for therapy of acromegaly. J Clin Endocrinol Metab, 2008; 93:2957-2968 
- Melmed, S., et al. Guidelines for Acromegaly Management: An Update. J Clin.Endocrinol Metab. 2009; 94:1509-1517.

- Scacchi, M., et al. Acromegaly. Pituitary. 2006; vol. 9 (4), pp. 297-303.

- Klein, I. Acromegaly and cancer. Annals of Internal Medicine. 1984; 101: 706-707.

- $\quad$ Klein, I., et al. Colonic polyps in patients with acromegaly. Annals of Internal Medicine. 1982; 97: 27 -30 .
- Ladas, S., et al. Does acromegaly really predispose to an increased prevalence of gastrointestinal tumors? Clin Endocrinol. 1994; 41:597-601

- Chobanien, S., et al. Skin tags as a marker for adenomatous polyps of the colon. Ann Intern Med. 1985; 103:892-893

- Jenkins, P., et al. Clinical perspecive: Acromegaly and cancer: A problem. J Clin Endocrinol and Metab. 2001; 86:2935-2945. 